\title{
NASA charts Earth observation strategy
}

[WASHINGTON] About 100 scientists gathered last month to begin charting a course for the next generation of low-cost Earth observation satellites, to follow the last of NASA's Earth Observing System (EOS) spacecraft scheduled for launch in 2002.

NASA called the invitation-only workshop to begin forming a consensus among scientists about missions to be flown from 2003 to 2010. The meeting was held near Washington DC and included observers from Europe, Japan, Canada and Brazil.

A panel led by Charles Kennel will report the results of the workshop by mid-October. Kennel was formerly the head of NASA's Earth science programme and is now director of the Scripps Institution of Oceanography in San Diego.

Before the meeting NASA received about

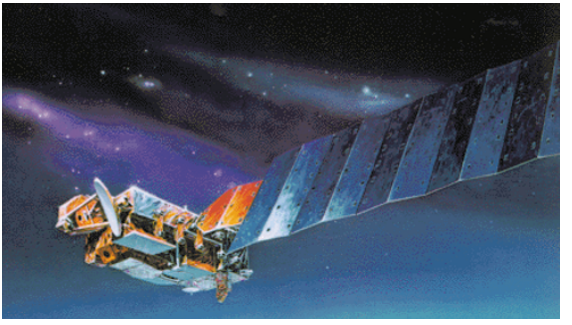

Eyes in space: an armada of Earth-observing satellites could be heading for orbit next century.

100 responses from scientists to a request for mission concepts. These were whittled down to the 23 most promising ideas by six discipline panels for climate studies, atmospheric chemistry, hydrology, oceans and ice, land cover and terrestrial ecosystems, and geodynamics and geology.

\section{Call for Europe-wide public health agency}

[PARIS] A group of international scientists meets for the first time this week, in Montpellier, France, to advocate the creation of a European equivalent of the US Centers for Disease Control (CDC). But political support for the proposal seems unlikely to be forthcoming at present, given the reluctance of the European Union's member states to cede sovereignty over public health policy to the European Commission.

The proposal for a European Centre for Control of Infectious Diseases (ECCID) is being championed by Michel Tibayrenc, from the Centre d'Etude sur le Polymorphisme des Micro-organismes in Montpellier. It has gained the support of several scientific organizations, including the European Society of Clinical Microbiology and Infectious Diseases. A committee of two dozen scientists has been established to promote ECCID, including Francisco Ayala from the University of California at Irvine; Dan Colley, head of the CDC's Division of Parasitic Diseases; and Koussay Dellagi, director general of the Pasteur Institute in Tunis.

The CDC was created in 1946 and has grown into a national disease prevention agency with nearly 7,000 staff. It runs large programmes for detecting and investigating health problems, carrying out research and control, training health professionals and promoting common policies in public health. An equivalent made up of the European Union and the countries of eastern Europe is needed in particular, argues Tibayrenc, to counter the threat of emerging and re-emerging diseases.

"In Europe, we have very poor coordination [of public health research and policy]," says Jean-Claude Piffaretti, chairman of the Swiss Society of Microbiology and a member of the new committee. He adds that a European CDC could create a much needed "big fundamental research effort in infectious diseases" and provide a focus for European efforts in developing countries.

The need for a more coordinated European effort in public health seems to attract a broad consensus among researchers and policymakers. But political support for a centralized public health agency is missing.

"Public health is politically very sensitive; we are talking about communicable diseases and big stakes in the economy of a nation," says one official from the European Commission's public health directorate. Individual member states would be reluctant to have a supranational body scrutinizing their national health systems, he says.

Indeed, the principle of a centralized organization, which was promoted by the European Parliament, was recently rejected by the Council of Ministers. Under the compromise agreement they reached, a network for the epidemiological surveillance and control of communicable diseases will link public health centres in member states with each other and with the commission's services.

The ECCID proposal draws attention to gaps in Europe's public health systems. Although the United Kingdom has a well developed Public Health Laboratory Service, Germany and other countries are widely considered to have poor public health systems, for example in surveillance, food safety and vaccination, compared with the United States.
Programme managers at the space agency produced an integrated "strawman" list of missions for consideration at the workshop. James Dodge of NASA's Earth science office says the list was only a "going-in position", meant to stimulate discussion, and is almost certain to change before the agency begins requesting detailed proposals for missions, perhaps as early as 2000

Those attending the workshop were given certain ground rules. Even the largest of the next generation of spacecraft are expected to cost 30 per cent less than the first generation of EOS missions. International and commercial partnerships are encouraged, and any proposal should assume cooperation with the National Polar-orbiting Operational Environmental Satellite System (NPOESS), the civilian-military weather satellite system that will see its first launch in 2009.

But many scientists are sceptical that the interests of operational weather forecasters and researchers will prove compatible in a joint programme. Also to be determined is what role new technologies will play in lowering mission costs while improving performance. NASA has begun an "incubator" programme to develop advanced instruments for Earth-viewing spacecraft, with the first grants to be announced this month.

The strawman list includes ideas for large spacecraft as well as small 'Earth probe' missions, and would balance long-term, systematic measurements of the kind conducted in the EOS series with more exploratory research satellites.

Among the concepts proposed for earliest development are a Land Cover/Land Use Inventory mission for launch in 2005 as a follow-on to Landsat 7; a Climate Variability and Trend satellite in 2006 to bridge the gap between the EOS-PM mission and the launch of the first NPOESS satellite; and a 2005 mission to monitor biological productivity of the oceans and land, which would bridge the gap between the EOS-AM spacecraft and the first NPOESS satellite.

NASA managers concede that a two-day meeting was hardly enough time to form a meaningful consensus on priorities. But Mark Abbott of Oregon State University says: “This isn't the final story - you'll see it evolve over the next year." NASA intends to have the Kennel group's recommendations reviewed by its own Earth science advisory committee and by the National Academy of Sciences.

Abbott, who chairs an advisory committee for EOS scientific payloads, says those attending the workshop represented a "much broader group" than just EOS investigators, and the "science questions were more sharply focused" because knowledge has advanced since the EOS missions were planned more than a decade ago.
Tony Reichhardt 